\title{
Reversibility and apparent tridimensionality
}

\author{
BRUCE O. BERGUM and LOIS E. FLAMM \\ College Station, Texas 77840
}

\begin{abstract}
Measures of perceived tridimensionality and reversal rates were taken on 101 undergraduate students to determine the relationship between these measures. Three examples each of the Necker and Mach reversible figures were employed. It was hypothesized that perceived tridimensionality would predict reversal rates at least as accurately as would objective measures of figural complexity, but without recourse to considerations of which "family" of geometric figures might be involved. The results confirmed the prediction. Perceived tridimensionality predicted reversal rates both within and across types of figures. It was suggested that the largely unexplored empirical relationships between these variables be more thoroughly investigated in parallel with studies of objective stimulus characteristics and perceived tridimensionality.
\end{abstract}

The objective definition of the stimulus for reversing figures has been a prime objective for several research inquiries in the past two decades. The underlying, if not always stated, assumption in this work is that reversals in the perception of bidimensional figures grow out of potential alternative figure-ground interpretations of these stimuli, and inherent in the concept of figureground is that of tridimensionality.

Hochberg and McAlister (1953) demonstrated that the tendency to perceive a figure as tridimensional is related to the amount of information contained in the figure, as defined by the number of lines and angles it contains; Hochberg and Brooks (1960) proposed that the relative apparent tridimensionality of a bidimensional figure would be a simple function of geometric complexity, defined as the number of angles contained in the figure, number of continuous line segments, and number of different angles divided by the total number of angles. The stimuli were subdivided into a number of "families," each consisting of several projections of a geometric figure. Their equations provided only for relative complexity within a given family, and thus subjects' responses were also limited to within-family ratings of tridimensionality. They found in a series of studies that the psychophysical judgments tended to support their equations.

Attneave $(1954,1957)$ and Attneave and Arnoult (1956) independently proposed an information interpretation similar to that of Hochberg and his colleagues. The "minimum" principle, or the tendency to see the object as simply as possible, was subsequently tested empirically by Attneave and Frost (1969), who found that their predictions of perceived apparent slant in bidimensional stimulus figures were supported by the judgments of the experimental subjects.

More recently, Vitz and Todd (1971) presented a

The second author is now at Bell Laboratories, Whippany Road, Whippany, New Jersey 07981. quantitative model of complexity based on number of trials to sample perceptual elements and number of axes of symmetry, from which they were able to predict the perceived tridimensionality demonstrated with the Hochberg and Brooks (1960) families of figures. They concluded that objectively measured "complexity" of bidimensional figures does predict tridimensional perceptions moderately well, but it suffers from inadequacies in specifying the conditions under which three- as opposed to two-dimensional complexity criteria should be applied.

While demonstrating the power of models to predict perceived tridimensionality, none of these studies attempted to examine directly the extent to which differences in figural complexity or informational characteristics actually result in differences in the frequency of associated figure reversals. However, a few empirical studies do suggest that apparent tridimensionality is an important determinant of figural stability.

In his most recent theorizing, Hochberg (1968) emphasized the importance of line intersection angles in tridimensional percept formation, but again provided no related reversal data to support the notion. Olson and Orbach (1966), however, presented empirical evidence that tends to partially support the Hochberg suggestion. Using various interpolated part-figures between presentations of the Necker cube, they found that the intersections of the cube were sufficient for reversals to occur, although not necessary. They also found that the oblique lines contributed nothing to the reversals.

Early studies of the relationship between reversal rates and complexity indicated lower rates for complex figures when compared with such "simple" figures as the Necker cube and the Mach figure (Donahue \& Griffitts, 1931; Porter, 1938). It should be noted, however, that some of the "complex" stimuli in these studies consisted of repeated patterns of Necker cube elements, and, in the Porter study, two figures representing animate objects. Ignoring the latter figures, the 
multiple Necker figures, as well as the Schroder staircase figure employed by Donahue and Griffitts, do present problems for the use of objective measures of complexity in predicting reversal rates and emphasize the subjective nature of deciding when to apply or not to bidimensional complexity measures, as noted by Vitz and Todd (1971). A complicating factor in the quantification of figural complexity is the matter of how to evaluate depictions of animate objects. It can be argued that the profiles represented in the Rubin face-vase figure are more complex in terms of number of lines, angles, and different angles than is the case for Necker cube drawings. In this sense they are more complex, and thus more tridimensional, than the cube. If tridimensionality is a factor in reversal rates, then the Rubin figure should logically elicit more reversals than the Necker cube. Yet the evidence is contrary to this prediction. Flamm and Bergum (1977) in fact found almost twice as many reversals for the Necker as compared with the Rubin (1921) figure. It is possible, of course, that once the threshold for perceived tridimensionality has been crossed, other factors such as familiarity or meaningfulness might enter into the determination of figural stability.

Whatever the relative merits of models for predicting perceived tridimensionality, the need continues for data relating apparent tridimensionality to figure reversals; the purpose of this experiment was to examine this relationship in terms of two stimulus sets drawn from the Hochberg and Brooks (1960) families. It was hypothesized that the number of reversals would increase as the degree of perceived tridimensionality increased and that direct measures of apparent tridimensionality would predict reversal rates more accurately than objective measures of stimulus complexity without recourse to considerations of which "family" of geometric figures might be involved.

\section{METHOD}

\section{Subjects}

One hundred and one undergraduate students at Texas A\&M University, 50 males and 51 females, volunteered to serve as subjects.

\section{Apparatus}

Two 35-mm slides depicting the Hill "young girl/old woman" figure and the Schroder staircase figure (Attneave, 1971) were employed to introduce the subjects to the concept of reversing figures. The slides were projected on a glass-beaded screen approximately $2.5 \times 2.5 \mathrm{~m}$ by means of a Sawyers $35-\mathrm{mm}$ rotary projector. An additional pair of slides was employed to illustrate the concept of apparent tridimensionality for two different line figures representing different versions of a pyramid. The figures are shown in the top portion of Figure 1. The lower portion of Figure 1 illustrates the six line figures employed, two at a time, in all combinations and permutations, in a final set of 30 experimental paired-comparison slides. The six figures represent three variations each of the Necker cube and the Mach figure (Attneave, 1971). The figures were drawn to represent three different-aspect angles of the two basic designs. The

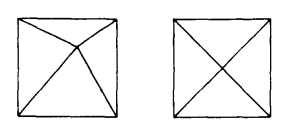

A
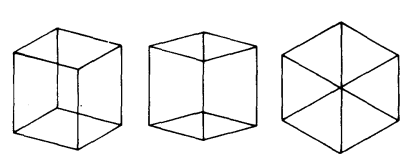
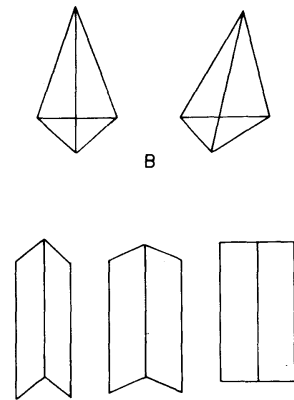

4

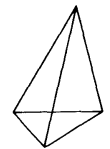

Figure 1. Demonstration figures and the six experimental figures.

Necker cube was drawn to range along a scale of figural symmetry from unsymmetrical, to half symmetrical, to perfectly symmetrical in the general sense described by Handel and Garner (1966) in their definition of dot figures. In the case of the Mach figure, the drawings varied along a scale of angularity of the two top and bottom line segments of the figure ranging from a downward deviation of $45 \mathrm{deg}$ from horizontal, to one of $30 \mathrm{deg}$ from horizontal, to a 0-deg deviation from horizontal.

Two booklets, approximately $11 \times 14 \mathrm{~cm}$ in size, were also employed. The first was a stimulus booklet consisting of a cover page and six additional pages, each displaying one of the six basic figures in a centered area of approximately $45 \times 45 \mathrm{~mm}$. At a point $10 \mathrm{~mm}$ from the right of center, and vertically centered on each figure, was a fixation $X$. The booklets were identical for all subjects except that they were assembled in six different random orders of stimulus figure presentation to control for possible sequential effects in viewing.

The second was a response booklet and included six blank, numbered pages on which to check reversals when seen by the subjects and a page with two columns of spaces in which to indicate, for each of the 30 paired-comparison slides, which member of the pair of depicted figures (left or right) appeared to the subject to be the more tridimensional.

Finally, a cassette tape-recorder/playback device was employed with a prerecorded tape for playback control of the slide presentation time intervals.

\section{Procedure}

The experiment was conducted in two parts, with all subjects participating as a group in both parts. In the first part, the assembled subjects were shown the two demonstration slides of reversing figures and given instructions on the nature of the reversal experience. The session did not formally begin until each subject acknowledged experiencing a reversal. They were then instructed to fixate on the " $\mathrm{X}$ " on each of the figures in the first booklet when given the signal, without looking away until told to stop, and to indicate by marking the response page each time they experienced a figure reversal. They were told to take a passive attitude and not be concerned if no change occurred (Ulrich \& Ammons, 1959). A 2-min viewing interval was employed throughout since reversal rate is known to increase in the first $2 \mathrm{~min}$ of passive viewing (Brown, 1955).

In the second part of the experiment, the subjects were shown the two demonstration slides of apparent tridimensionality and how they might be perceived as representations of solid objects. They were then instructed to indicate in the response booklet which member of the pair of figures presented on each paired-comparison slide appeared to be the more tridimensional. They were instructed to make a choice for every pair, regardless of how certain they felt about their choices. The 30 slides were presented in predetermined random order at 
5-sec intervals. Slide numbers were called aloud to assure that the responses were correctly recorded by the subjects.

\section{RESULTS}

The data were analyzed, first, for the perceived tridimensionality of the six figures and, second, in terms of the number of figure reversals associated with each of the figures. In both cases, a rank order was determined for the six stimuli for each subject in terms both of his frequency of paired-comparison choices for each figure and of the number of reversals experienced for each stimulus. These data were analyzed by means of Friedman (1937) nonparametric tests.

Table 1 presents the data in terms of the mean ranks for all subjects for each stimulus figure, for both the apparent tridimensionality of the stimuli and the degree of reversing experienced by the subjects.

As indicated in Table 1, both sets of figures were ordered according to the predictions of perceived tridimensionality, with the Necker family of figures demonstrating in all cases a generally more apparent tridimensionality than was the case for the Mach figures. The Friedman test indicated that these differences were significant $\left[\chi^{2}(5)=175.19, p<.001\right]$. The least symmetrical Necker cube was ranked highest and the most symmetrical Mach figure was ranked lowest in perceived tridimensionality.

The results also indicate that the stimulus figures were ordered according to the predictions for the relative frequency of reversals, with the least symmetrical Necker cube resulting in the greatest relative number of reversals and the most symmetrical Mach figure yielding the fewest reversals of perspective. Again, the Friedman analysis was significant $\left[\chi^{2}(5)=96.87\right.$, $\mathrm{p}<.001]$.

The results tend to support the hypothesis that figural reversibility is directly related to the apparent tridimensionality of bidimensional figures. Of some additional interest is the fact that, while there was a gradual decrease in both apparent tridimensionality and relative reversal rates for Figures 1-5, a dramatic change occurred between Figures 5 and 6 , the latter providing virtually none of the common cues to solidity.

\section{DISCUSSION}

Reversal rates were found to relate directly to perceived

Table 1

Mean Ranks for the Six Figures in Terms of Their Perceived Tridimensionality and Relative Reversal Rates

\begin{tabular}{lcccccc}
\hline & \multicolumn{6}{c}{ Figure } \\
\cline { 2 - 7 } & 1 & 2 & 3 & 4 & 5 & 6 \\
\hline Perceived 3-D & 2.73 & 2.94 & 2.99 & 3.24 & 3.37 & 5.76 \\
Reversals & 2.67 & 2.82 & 2.93 & 3.10 & 3.29 & 5.72 \\
\hline
\end{tabular}

tridimensionality, regardless of figure "family" considerations. The Necker cube was seen as generally more amenable to tridimensional interpretation than was the Mach figure, yielding more reversals; within these families, those versions seen as more tridimensional also yielded more reversals. Of particular interest were the relative magnitudes, as expressed by mean ranks, of the six figures along both dimensions. Both in the case of perceived tridimensionality and reversals, Figure 6 yielded very large, and essentially the same magnitude, deviations on both dimensions.

Predictions based upon an objective measurement of complexity would have yielded grossly similar results perhaps, but with substantial distortions in the specifics of the relationships. For example, number of turns, found by Attneave (1957) to be an important determinant of complexity, is unchanged for each of the Mach figures. Based also upon the Hochberg (1968) factors of number of angles, different angles, and lines, the two families would have differed more than they actually did and, in the case of the Mach figures, the extreme values for Figure 6 would not have been predicted.

Nonlinear relationships between physically described stimulus dimensions and psychological response dimensions are characteristic in psychophysical work (Stevens, 1964), and the demonstration of additional instances should not be surprising. If perceived tridimensionality is to be used as an explanatory mechanism for figural stability phenomena, one profitable course of action might be to begin with the demonstration of a variety of empirically derived relationships between perceived tridimensionality and reversal rates, followed then by formulations of the specific relationships between degree of perceived tridimensionality and objectively described stimulus characteristics.

\section{REFERENCES}

Attneave, F. Some informational aspects of visual perception. Psychological Review, 1954, 61, 183-193.

Attneave, F. Physical determinants of the judged complexity of shapes. Journal of Experimental Psychology, 1957, 53, 221-227.

Attneave, F. Multistability in perception. Scientific American, $1971,225,62-71$.

Attneave, F., \& Arnoult, M. D. The quantitative study of shape and pattern perception. Psychological Bulletin, 1956, 6, 452-471.

Atrneave, F., \& Frost, R. The determination of perceived tridimensional orientation by minimum criteria. Perception \& Psychophysics, 1969, 6, 391-396.

Brown, K. T. Rate of apparent change in a dynamic ambiguous figure as a function of observation time. American Journal of Psychology, 1955, 68, 358-371.

Donahue, W. T., \& GriffitTs, C. H. The influence of complexity on the fluctuations of the illusions of reversible perspective. American Journal of Psychology, 1931, 43, 613-617.

Flamm, L. E., \& Bergum, B. Reversible perspective figures and eye movements. Perceptual and Motor Skills, 1977, 44, 10151019.

Friedman, M. The use of ranks to avoid the assumption of normality implicit in the analysis of variance. Journal of the American Statistical Association, 1937, 32, 674-701.

HANDEl, S., \& Garne R, W. R. The structure of visual pattern associates and pattern goodness. Perception \& Psychophysics, $1966,1,33-38$

Hochbe RG, J. In the mind's eye. In R. N. Haber (Ed.), Contemporary theory and research in visual perception. New York: Holt, Rinehart, \& Winston, 1968.

HochberG, J., \& Brooks, V. The psychophysics of form: Reversible-perspective drawings of spatial objects. American Journal of Psychology, 1960, 73, 337-354. 
Hochberg, J., \& McAlister, E. A quantitative approach to figural “goodness." Journal of Experimental Psychology, 1953, 46, 361-364.

Olson, R., \& Orbach, J. Reversibility of the Necker cube: VIII. Parts of the figure contributing to the perception of reversals. Perceptual and Motor Skills, 1966, 22, 623-629.

Porte R, E. L. H. Factors in the fluctuation of fifteen ambiguous phenomena. Psychological Record, 1938, 2, 231-253.

RUBIN, E. Visuelle wahrgenommene Figuren. Copenhagen: Guldendalske, 1921.
Stevens, S. S. Concerning the psychophysical power law. Quarterly Journal of Experimental Psychology, 1964, 16, 383-385.

UlRich, P., \& Ammons, R. B. Voluntary control over perceived dimensionality (perspective of three-dimensional objects). Proceedings of the Montana Academy of Sciences, 1959, 19, 169-173.

Vitz, P. C., \& TodD, T. C. A model of the perception of simple geometric figures. Psychological Review, 1971, 78, 207-228.

(Received for publication June 13, 1979.) 